\title{
Light variations due to the line-driven wind instability and wind blanketing in $\mathrm{O}$ stars
}

\author{
J. Krtička ${ }^{1}$ and A. Feldmeier ${ }^{2}$ \\ 1 Ústav teoretické fyziky a astrofyziky, Masarykova univerzita, Kotlářská 2, 61137 Brno, Czech Republic \\ e-mail: krticka@physics.muni.cz \\ 2 Institut für Physik und Astronomie, Universität Potsdam, Karl-Liebknecht-Straße 24/25, 14476 Potsdam-Golm, Germany \\ Received 21 July 2017 / 28 June 2018
}

\begin{abstract}
A small fraction of the radiative flux emitted by hot stars is absorbed by their winds and redistributed towards longer wavelengths. This effect, which leads also to the heating of the stellar photosphere, is termed wind blanketing. For stars with variable winds, the effect of wind blanketing may lead to the photometric variability. We have studied the consequences of line driven wind instability and wind blanketing for the light variability of $\mathrm{O}$ stars. We combined the results of wind hydrodynamic simulations and of global wind models to predict the light variability of hot stars due to the wind blanketing and instability. The wind instability causes stochastic light variability with amplitude of the order of tens of millimagnitudes and a typical timescale of the order of hours for spatially coherent wind structure. The amplitude is of the order of millimagnitudes when assuming that the wind consists of large number of independent concentric cones. The variability with such amplitude is observable using present space borne photometers. We show that the simulated light curve is similar to the light curves of $\mathrm{O}$ stars obtained using BRITE and CoRoT satellites.
\end{abstract}

Key words. stars: winds, outflows - stars: mass-loss - stars: early-type - stars: variables: general - hydrodynamics

\section{Introduction}

The advent of photometers on board dedicated spacecrafts like Kepler, CoRoT, MOST, and BRITE revolutionized the field of astronomical photometry. The space-borne photometers provide not only light curves with unprecedented coverage, but they also lead to a significant improvement of the precision of measurements. As a result, stars that were deemed constant were found to be variable leading to discoveries of new types of variable stars.

Single O stars are a typical examples of stars for which no variability was expected, yet that still show low-amplitude light variations. For example, significant low-frequency variability was detected in Kepler photometry of a blue supergiant HD 188209 and was attributed to the gravity waves (Aerts et al. 2017). A dedicated CoRoT observing run focused on the young open cluster NGC 2244 detected stochastic variations in O stars, which were attributed to the red noise that is possibly due to the subsurface convection (Blomme et al. 2011) and multiple frequencies due to stellar oscillations (Degroote et al. 2010; Briquet et al. 2011; Mahy et al. 2011). Similar variations were also found after the subtraction of binary light curve in $\delta$ Ori Aa (Pablo et al. 2015), in early-B supergiant HD 2905 (Simón-Díaz et al. 2018), and in $\zeta$ Pup (Ramiaramanantsoa et al. 2018).

Low-amplitude light variability in $\mathrm{O}$ stars may be connected to the line-driven wind instability. As a result of the Doppler effect, wind driving by multiple lines in hot stars is unstable (Lucy \& Solomon 1970; Owocki \& Rybicki 1984). The numerical simulations have shown that the line-driven wind instability leads to the variability of the wind density, velocity, and mass-loss rate on a typical scale of hours (Owocki et al. 1988; Feldmeier et al. 1997). Due to supersonic nature of hot star winds, the wind instability leads also to appearance of wind shocks and the wind X-ray emission (Feldmeier et al. 1997). The line-driven wind instability is likely connected with clumping, which affects observed wind spectral features (e.g. Sundqvist et al. 2010, 2011; Šurlan et al. 2012, 2013; Shenar et al. 2015) and therefore affects the wind mass-loss rate estimates.

Wind instabilities are self-initiating (Owocki et al. 1988), but may be also initiated and modulated by photospheric motions (Feldmeier et al. 1997). Consequently, the instabilities may be connected with photospheric turbulence whose presence affects, for example, the widths of photospheric lines (Aerts et al. 2009; Cantiello et al. 2009; Jiang et al. 2015).

Part of the flux emerging from the photospheres of stars is absorbed by the wind and emitted back to the stellar photosphere. The backwards emission causes heating of the photosphere especially at low optical depths and also leads to redistribution of the flux mainly from the short-wavelength part of spectrum to longer wavelengths. This effect is termed wind blanketing (Abbott \& Hummer 1985) and is important for obtaining precise effective temperatures of hot stars with strong winds (Bohannan et al. 1986; Crowther et al. 2002; Martins et al. 2005). The wind blanketing redistributes the emergent flux in dependence of wind opacity, and amount of redistribution depends on the wind mass-loss rate. Therefore, wind blanketing does not lead to any light variability for fixed massloss rate.

On the other hand, if the mass-loss rate is variable, then the wind blanketing is modulated by the mass-loss rate, resulting in the photometric variability. For example, in magnetic hot stars the wind mass flux depends on the tilt of the magnetic field (Owocki \& ud-Doula 2004). Therefore, the strength of the wind 
blanketing varies across the stellar surface, which, due to stellar rotation, leads to the photometric variability of magnetic $\mathrm{O}$ stars. This effect can partly explain the photometric light curve of HD 191612 (Krtička 2016).

The line-driven wind instability is another process that leads to the mass-loss rate variation (Owocki et al. 1988; Feldmeier et al. 1997; Runacres \& Owocki 2002). Therefore, also the line-driven wind instability causes photometric variability in hot stars. This can be connected with the low-amplitude variability observed in O stars. To study this possibility, we used hydrodynamical simulation of Feldmeier et al. (1997) to derive the mass-loss rate variation and the relation between the massloss rate and photometric flux (Krtička 2016) to predict the light variability.

\section{Modelling of the light variability: spherically symmetric case}

The calculation of the light variability of hot stars due to the linedriven wind instability is a very complex problem. In general, 3D time-dependent hydrodynamical simulations of the stellar wind are required to obtain the density and velocity structure of the circumstellar environment of hot stars and its variability. Such simulations have to be coupled to a global (unified) 3D wind model that includes the influence of the wind on the stellar photosphere (accounting for wind blanketing) and that is able to solve the radiative transfer equation for moving media whose ionization and excitation state is out of equilibrium. There is no such code that would be able to treat the whole complex problem. Therefore, we used existing wind simulations and global wind models and combine their output to derive the expected light variations in our stars. Due to the simplifications involved, we have not focussed on a particular star or stellar type, but instead we aim to obtain general magnitude of the effect and its properties.

\subsection{Hydrodynamical simulations}

The hydrodynamical simulations we have employed here were described by Feldmeier et al. (1997) in detail. The simulations solved continuity, momentum, and energy equation for a spherically symmetric non-rotating wind flow. The simulations were performed using the smooth source function method (Owocki 1991; Owocki \& Puls 1996). The hydrodynamical part of the code is based on a standard van Leer solver. The simulations use staggered mesh, operator splitting of advection and source terms, advection terms in a conservative form using van Leer's (1977) monotonic derivative as an optimised compromise between stability and accuracy, Richtmyer artificial viscosity, and non-reflecting boundary conditions (Hedstrom 1979; Thompson 1987, 1990). A turbulent variation of the velocity at a level of roughly one third of the sound speed was introduced as seed perturbation for unstable growth at the wind base.

The hydrodynamical simulations were performed for specific stellar parameters corresponding to $\zeta$ Ori $\mathrm{A}$, that is the effective temperature $T_{\text {eff }}=31500 \mathrm{~K}$, radius $R_{*}=24 R_{\odot}$, and mass $M=34 M_{\odot}$, and yielded the mean mass-loss rate $\dot{M}_{0}=$ $3 \times 10^{-6} M_{\odot} \mathrm{yr}^{-1}$. The simulations are robust against changes in stellar parameters. Consequently, the relative variations of wind density derived for such specific parameters are also applicable to other $\mathrm{O}$ stars.

The calculation of the wind blanketing directly from the hydrodynamic simulations would require solution of radiative transfer and kinetic equilibrium equations (also called NLTE

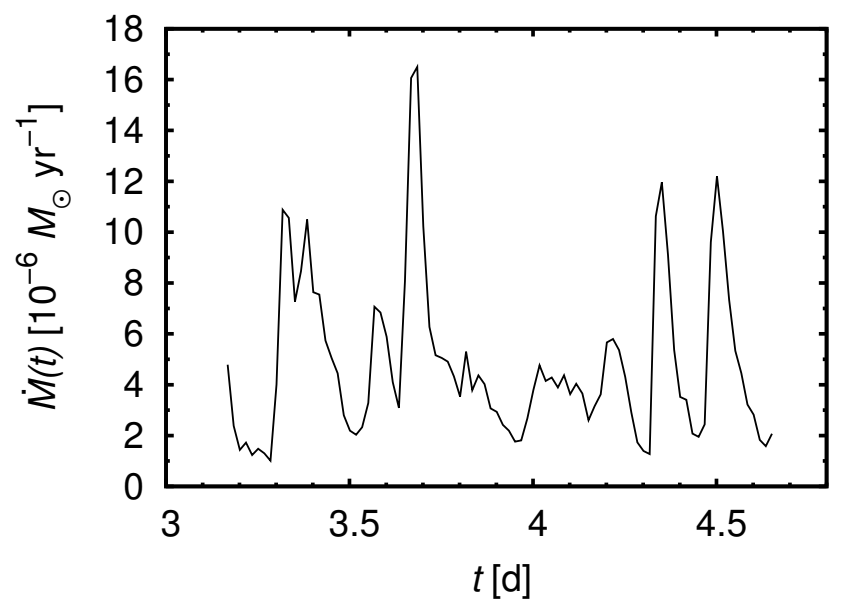

Fig. 1. Mass-loss rate from the numerical simulations averaged over $r \in\left[1.01 R_{*}, 1.02 R_{*}\right]$ (see Eq. (1)).

equations) for non-monotonic velocity law. To our knowledge, there is no such code that is able to treat this problem in its full complexity. Therefore, to make the problem tractable, we calculate a mean value of the mass-loss rate in the region close to the star, where the wind blanketing originates,

$\dot{M}(t)=\frac{4 \pi}{r_{2}-r_{1}} \int_{r_{1}}^{r_{2}} r^{2} \rho|v| \mathrm{d} r$,

where $\rho$ and $v$ are the wind density and radial velocity in particular time. Most of the flux in the optical domain is emitted from the region below the sonic point, which typically appears at radii $1.01-1.02 R_{*}$ in the numerical simulations. Therefore, we selected $r_{1}=1.01 R_{*}$ and $r_{2}=1.02 R_{*}$. However, the final light curve does not significantly depend on the choice of $r_{1}$ and $r_{2}$. As a result of this choice, in Eq. (1) we average over 50 grid points of hydrodynamical simulation. A plot of $\dot{M}(t)$ is given in Fig. 1.

\subsection{Global wind models}

The variations of the emergent flux with wind mass-loss rate were derived from spherically symmetric stationary METUJE global wind models (Krtička \& Kubát 2017). The code calculates the wind model in a global (unified) approach that integrates the description of the hydrostatic photosphere and supersonic wind. The model solves the radiative transfer equation in the co-moving frame (CMF) with opacities and emissivities calculated using occupation numbers derived from the kinetic equilibrium (NLTE) equations. For given stellar parameters, the model enables us to predict the radial dependence of the density, velocity, and temperature from hydrodynamical equations and to derive the wind mass-loss rate $\dot{M}$ and the stellar emergent flux that accounts for the wind blanketing.

For our modelling we assumed a typical parameters of $\mathrm{O}$ stars corresponding to HD $191612, T_{\mathrm{eff}}=36000 \mathrm{~K}, R_{*}=$ $14.1 R_{\odot}$, and $M=29.2 M_{\odot}$. To model the dependence of the emergent flux on the mass-loss rate with fixed stellar parameters, we artificially scaled the radiative force in the wind. This yields a series of wind models and emergent fluxes $F(\lambda, \dot{M})$ parameterized by the wind mass-loss rate. Because the amount of the flux redistributed by the wind depends on the effective temperature, we expect slightly different dependence for stars with different temperature. However, because we are concerned with 


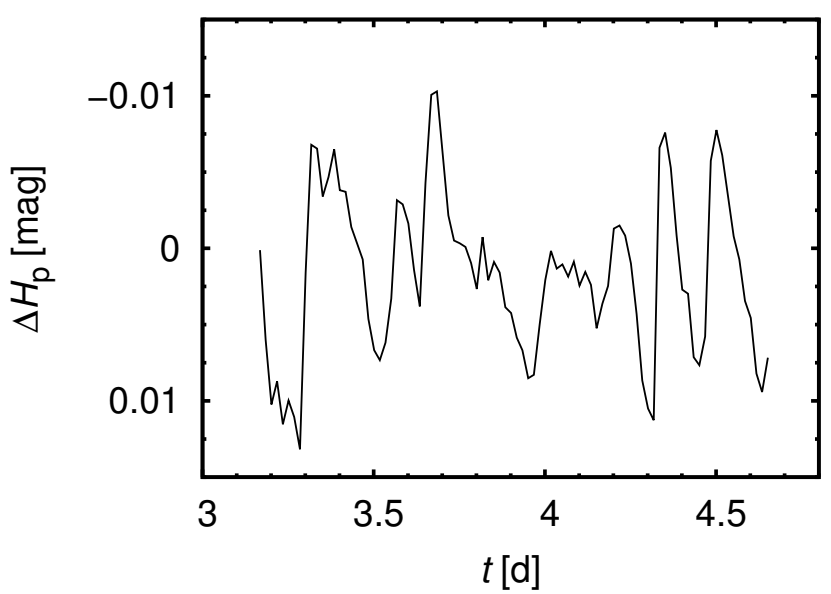

Fig. 2. Light curve calculated using Eq. (2) for the mass-loss rate variations plotted in Fig. 1.

typical light variations, we limited our study to just one star. On the other hand, such selection affects only the amplitude of the light curve. The preliminary results derived for 300-1 model of Krtička \& Kubát (2017, the model has parameters close to that used in hydrodynamical simulations) indicate that the amplitude of the variability due to the wind blanketing does not significantly depend on stellar parameters.

The light variability is derived from a relation between the $H_{\mathrm{p}}$ magnitudes and mass-loss rate (Krtička 2016, Eq. (2))

$\Delta H_{\mathrm{p}}(t)=-2.5 \log (e) \frac{\Delta F}{F_{0}} \log \left(\frac{\dot{M}(t)}{\dot{M}_{0}}\right)$,

where $\Delta F=1.6 \times 10^{7} \mathrm{erg} \mathrm{s}^{-1} \mathrm{~cm}^{-2}, F_{0}=9.0 \times 10^{8} \mathrm{erg} \mathrm{s}^{-1} \mathrm{~cm}^{-2}$ is the emergent flux for a reference mass-loss rate, and $\dot{M}_{0}$ is the mean mass-loss rate. The relation was derived for HD 191612 parameters using METUJE global wind models (Krtička \& Kubát 2017).

\subsection{Calculated light curve}

The light curve calculated using Eq. (2) for the mass-loss rate variations plotted in Fig. 1 is given in Fig. 2. The amplitude of the light curve is of the order of $0.01 \mathrm{mag}$ and the typical timescale of the light variability (hours) is the same as the timescale of the variability of the mass-loss rates as derived from the simulations.

The amplitude of the light curve is proportional to the massloss rate variations, which depend on the surface perturbations. The light variations are therefore stronger for stronger base perturbations. The wind structure is triggered by the photospheric perturbations, which therefore determine the time scale of the variability.

\section{The case without spherical symmetry}

Within a spherical symmetry we assumed that mass-loss rate variations are coherent across the stellar surface. This assumption is highly unrealistic for real wind variations. However, the coherence length in the horizontal direction is unclear. A typical patch size of the instability generated structure is of the order of degrees, as inferred from observations (e.g. Dessart \& Owocki 2002) and the typical number of clumps in the observed part of the wind is of the order of $10^{3}-10^{5}$ (Davies et al. 2007; Oskinova et al. 2007; Nazé et al. 2013; Šurlan et al. 2013).
To account for the deviation from spherical symmetry, we followed the approach of Dessart \& Owocki (2002) and Feldmeier et al. (2003) and assumed that the stellar wind consists of $N$ concentric cones. We assumed that each of these cones is independent, and that the mass-loss rate from the $i$ th cone with stellar surface cross-section $\Omega_{i} R_{*}^{2}$ at time $t$ is

$\dot{M}_{i}(t)=\frac{\Omega_{i}}{4 \pi} \dot{M}\left(t+\Delta t_{i}\right)$

where $\Delta t_{i}$ is random for each $i$ and $\dot{M}(t)$ is given by Eq. (1). Moreover, we assumed that $\dot{M}(t)$ varies periodically with the period given by the time extent of available simulations (which is about $1.5 \mathrm{~d}$, see Fig. 1).

The cones were selected in such a way that $\Omega_{i}$ is roughly the same for all cones. We divided the visible stellar surface into elements bounded by a specified number of concentric rings. The first element that directly faces the observer is assumed to specify just one cone. The number of cones specified by other rings is selected in such a way that solid angle set by the cones is roughly equal to solid angle of the first cone. The number of cones is therefore a function of the number of rings. Because we specify the number of latitudinal rings, we cannot calculate the light curves for an arbitrary number of cones.

The magnitude difference between the observed flux $f_{H_{\mathrm{p}}}(t)$ at a given time and the reference flux $f_{H_{\mathrm{p}}}^{\text {ref }}$ in passband $H_{\mathrm{p}}$ is defined as

$\Delta H_{\mathrm{p}}(t)=-2.5 \log \left(\frac{f_{H_{\mathrm{p}}}(t)}{f_{H_{\mathrm{p}}}^{\text {ref }}}\right)$.

The reference flux is obtained under the condition that the mean magnitude difference over the simulated light curve is zero. The radiative flux in a passband $H_{\mathrm{p}}$ at the distance $D$ from the spherical star is (Hubeny \& Mihalas 2014)

$f_{H_{\mathrm{p}}}(t)=\left(\frac{R_{*}}{D}\right)^{2} \int_{\substack{\text { visible } \\ \text { surface }}} I_{H_{\mathrm{p}}}(\theta, \Omega, t) \cos \theta \mathrm{d} \Omega$.

The intensity $I_{H_{\mathrm{p}}}(\theta, \Omega, t)$, at angle $\theta$ with respect to the normal to the surface was obtained at each surface point with spherical coordinates $\Omega$ from the emergent flux taking into account the limb darkening $u_{H_{\mathrm{p}}}(\theta)$

$I_{H_{\mathrm{p}}}(\theta, \Omega, t)=u_{H_{\mathrm{p}}}(\theta) I_{H_{\mathrm{p}}}(\theta=0, \dot{M}(\Omega, t))=\frac{u_{H_{\mathrm{p}}}(\theta)}{\left\langle u_{H_{\mathrm{p}}}\right\rangle} F_{H_{\mathrm{p}}}(\dot{M}(\Omega, t))$,

with limb darkening coefficients from Reeve \& Howarth (2016). Here $\left\langle u_{H_{\mathrm{p}}}\right\rangle=2 \pi \int_{0}^{\pi / 2} u_{H_{\mathrm{p}}}(\theta) \cos \theta \sin \theta \mathrm{d} \theta$. The emergent flux $F_{H_{\mathrm{p}}}(\dot{M}(\Omega, t))$ is obtained from Eq. (2) modified for fluxes, and the mass-loss rate $\dot{M}(\Omega, t)$ is given by Eq. (3) for a cone that corresponds to given coordinates $\Omega$.

The resulting light curves are given in Fig. 3. From this figure it follows that the amplitude of the light variability due to wind instability quickly decreases with the number of cones. On the other hand, even with a relatively large number of cones, the light variability should be detectable with satellite photometry. 


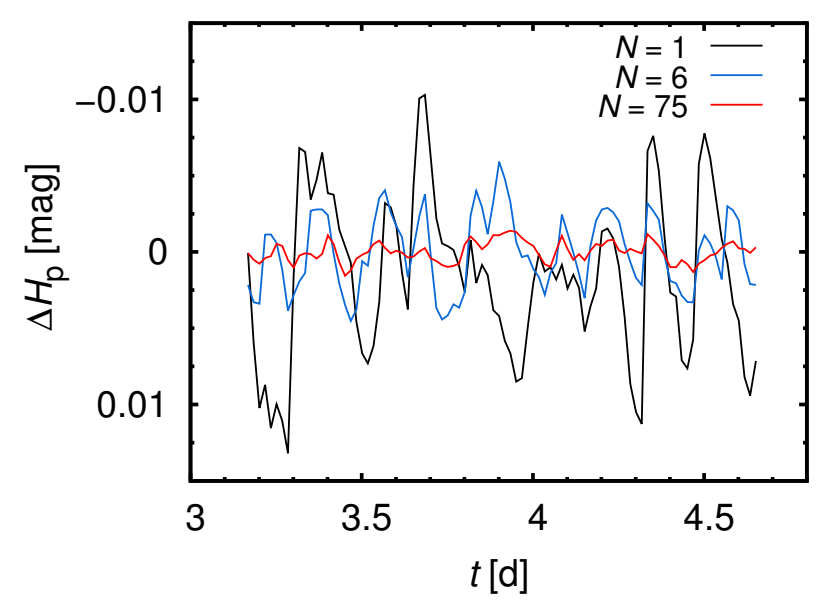

Fig. 3. Light curve calculated using Eq. (4) for different number $N$ of concentric cones.

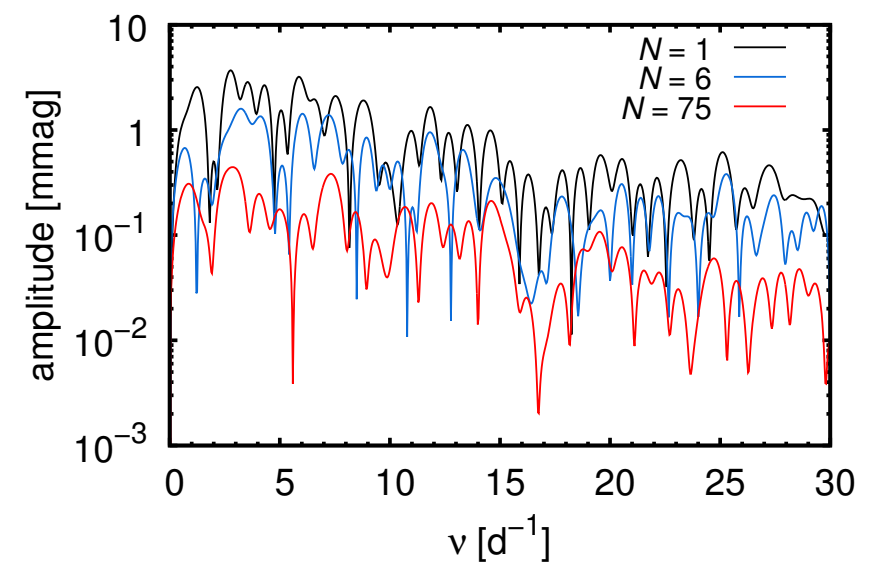

Fig. 4. Fourier analysis of the light curves given in Fig. 3.

\section{Comparison with observations}

We used Period04 (Lenz \& Breger 2005) to analyse the simulated light curves (see Fig. 4). The general shape of the Fourier spectrum does not significantly depend on the number of cones used to calculate the light curve. The Fourier spectrum shows maximum at the frequency of about $3 \mathrm{~d}^{-1}$ which corresponds to the characteristic times scale of the variability of the mass-loss rate. The location of the maximum peak is nearly independent of the number of assumed cones. There are further peaks at higher frequencies. An additional low-frequency peak at about $1 \mathrm{~d}^{-1}$ is most likely connected with the length of available simulations.

Due to a stochastic nature of the light curves, a direct comparison of the simulated and observed light curves is not meaningful. On the other hand, it is possible to compute the Fourier spectrum of the light curves, fit the spectrum by some phenomenological model, and compare the fit parameters derived for observed and simulated light curves. Similar techniques are used to study time series in various contexts, for example, light curves of X-ray binaries (Burderi et al. 1993), solar flare data (Threlfall et al. 2017), or to search for granulation in Cepheids (Derekas et al. 2017).

To compare the theoretical light curves with observations, we fitted the Fourier spectrum $A(v)$ in Fig. 4 by a polynomial

$\log \left(\frac{A(v)}{1 \mathrm{mmag}}\right)=a\left(\frac{v}{1 \mathrm{~d}^{-1}}\right)+b$.
Table 1. Parameters of the fit Eq. (7) of the Fourier spectrum.

\begin{tabular}{llcc}
\hline \hline Parameter & & $a$ & $b$ \\
\hline Simulation & $N=1$ & -0.030 & -2.87 \\
& $N=6$ & -0.027 & -3.25 \\
& $N=75$ & -0.034 & -3.71 \\
\hline Star & HD 37128 & -0.11 & -2.93 \\
& HD 46150 & -0.035 & -4.26 \\
& HD 46223 & -0.037 & -3.94 \\
& HD 46966 & -0.032 & -4.49 \\
\hline
\end{tabular}

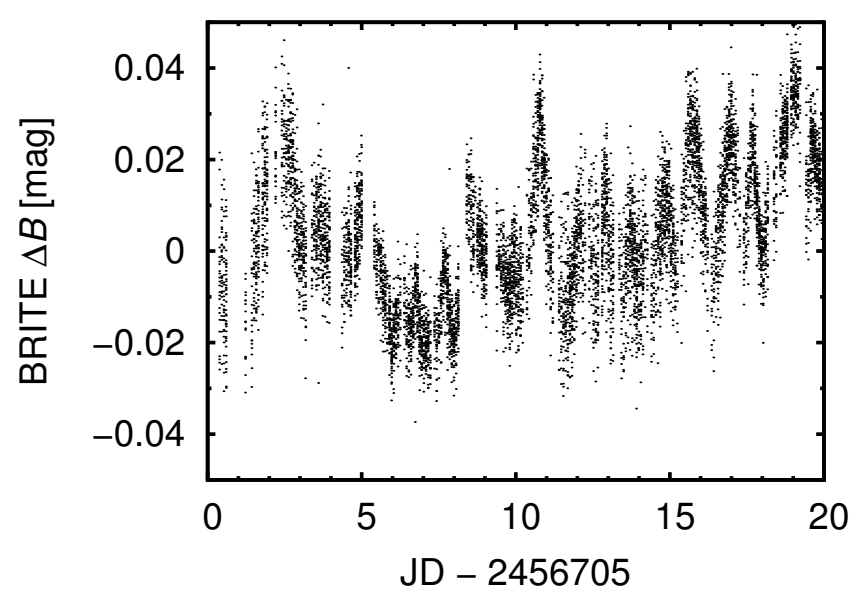

Fig. 5. Portion of the light curve of $\epsilon$ Ori derived using the BRITE satellite. Difference between the actual and mean magnitudes is plotted.

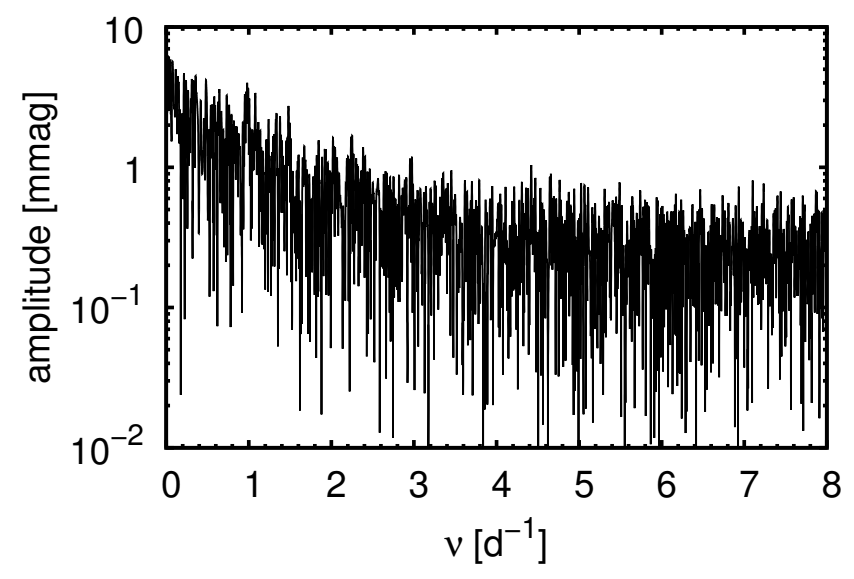

Fig. 6. Fourier spectrum of the light curve of $\epsilon$ Ori.

The fit parameters given in Table 1 demonstrate that the shape of the Fourier spectrum (given by $a$ parameter) does not depend on the assumed number of cones, while the amplitude of peaks (described by $b$ ) depends on $N$. The fit of results from Table 1 shows that the parameter $b$ varies with $N$ on average as

$b=-0.45 \log N-2.88$.

The simulated light curve can be compared, for example, with the light curve of $\epsilon$ Ori (HD 37128, B0Ia) derived using the BRITE satellites. The BRITE-Constellation consists of six satellites working in blue and red domains of visible spectra (Weiss et al. 2014). The satellites provide high-precision photometry of bright objects including chemically peculiar stars, 

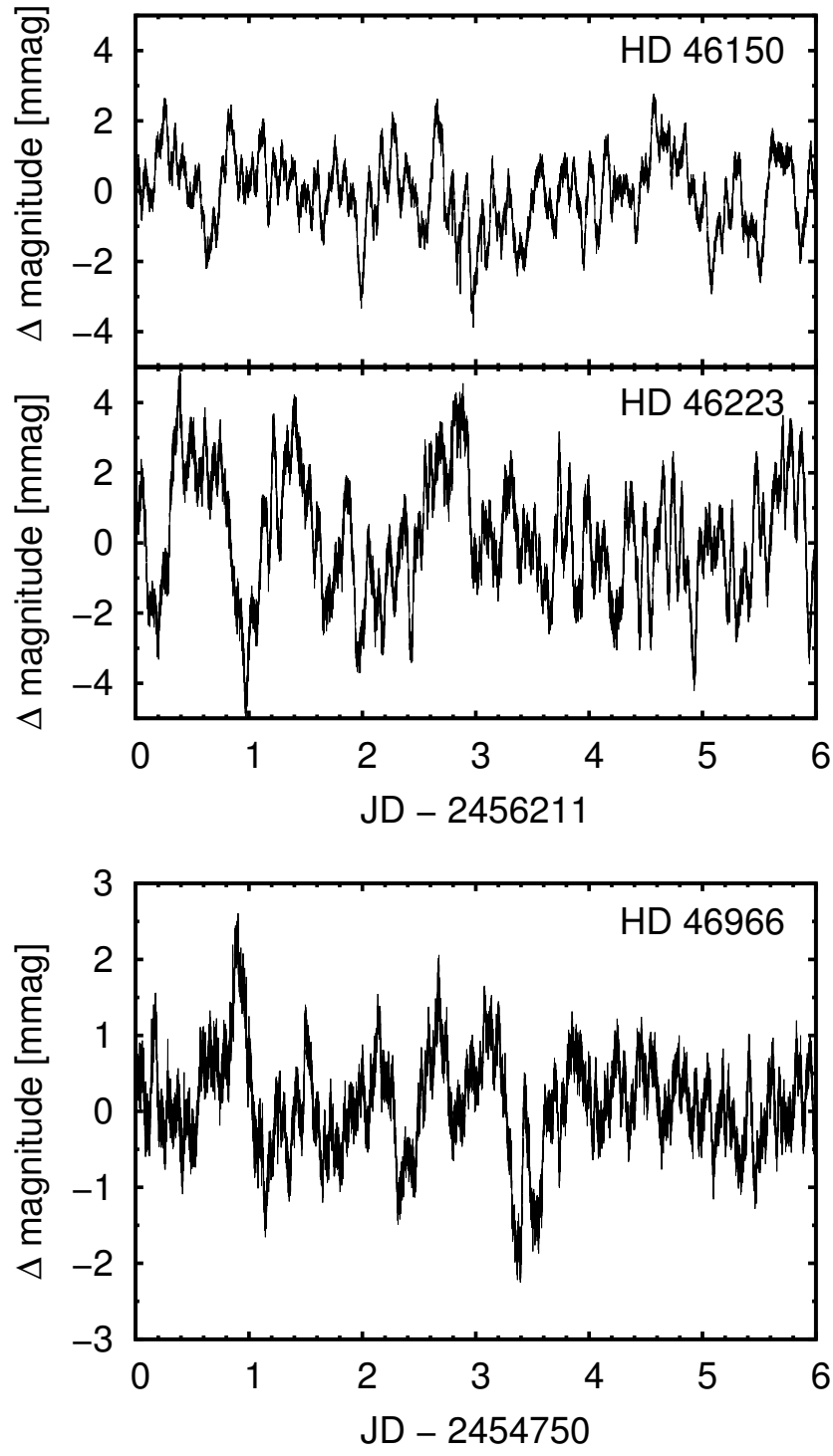

Fig. 7. Portion of the light curve of $\mathrm{O}$ stars derived using the CoRoT satellite. Difference between the actual and mean magnitudes is plotted.

pulsating stars, and Be stars (Baade et al. 2016; Weiss et al. 2016; Daszyńska-Daszkiewicz et al. 2017; Handler et al. 2017). The data were downloaded from the BRITE public data archive ${ }^{1}$. We used observations of $\epsilon$ Ori obtained by BAb (BRITEAustria) satellite between JD 2456628 and 2456734. We used observations in a blue filter which covers the wavelength range of 390-460 nm (Kaiser et al. 2008). The observed differential light curve shows complex variability with amplitude comparable to the simulated light variations in Fig. 2 and a typical timescale of about $1 \mathrm{~d}$ (see Fig. 5 and also David-Uraz et al. 2017a). A corresponding peak appears also in the Fourier spectrum in Fig. 6. Consequently, while the time scale of the variability is comparable to that derived from simulations, the interpretation of the amplitude of the observed variability would require either a coherent wind variability or a very strong base perturbation.

The inspection of the BRITE archive revealed that a similar type of variability is also likely present in two additional stars $\zeta$ Ori A (O9.2Ib, HD 37742, HR 1948) and J Pup (B0.5Ib,

\footnotetext{
1 https://brite.camk.edu.pl/pub/index.html
}

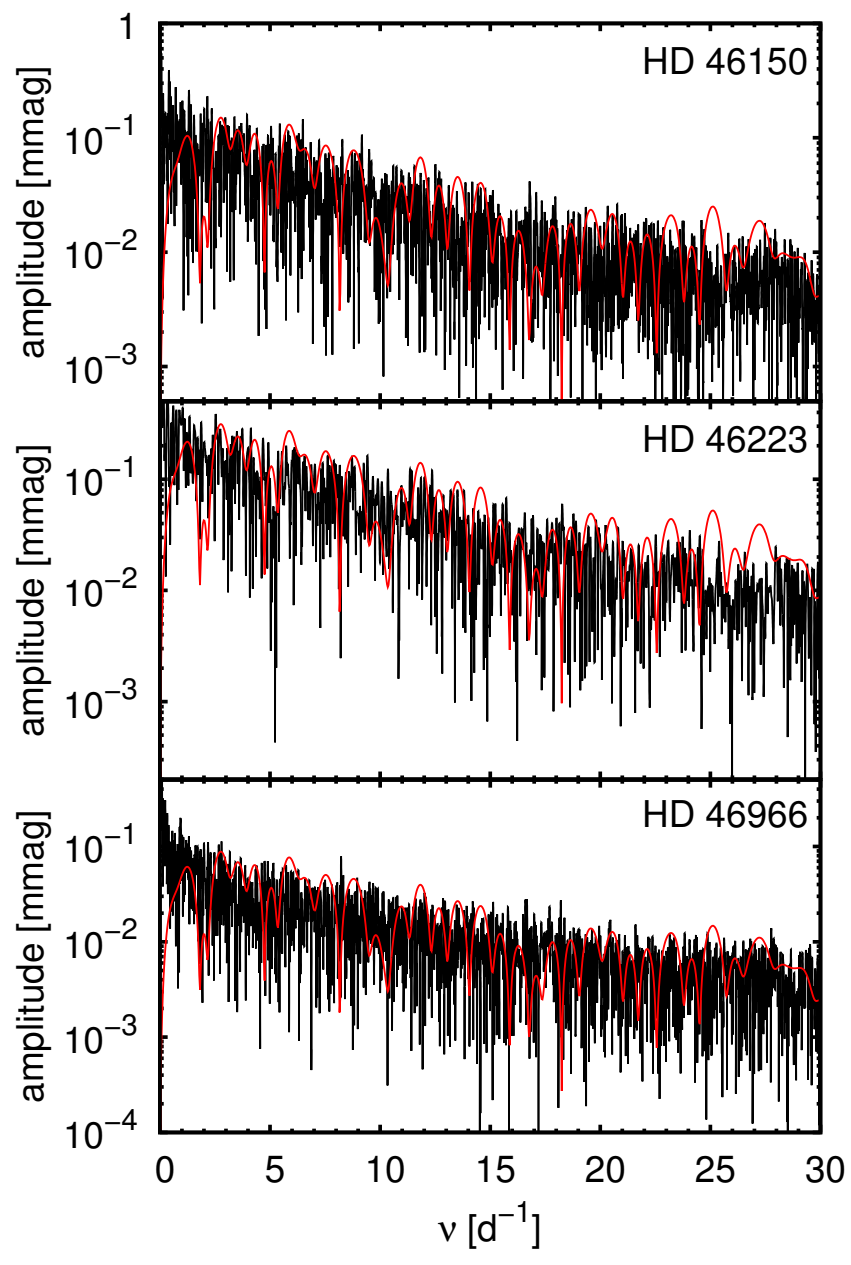

Fig. 8. Comparison of the Fourier spectra of light curves given in Fig. 7 (black curves) with Fourier spectrum of the theoretical light curve for $N=1$ (red curves). The theoretical spectrum was scaled by a factor of $10^{b}$ taken from Table 1 accounting for $N>1$. For the analysis we used the CoRoT light curves secured between JD 2456203 and 2456234 for HD 46150 and HD 46223 and between JD 2454748 and 2454783 for HD 46966

HD 64760, HR 3090). The light variations of these stars have roughly the same amplitude and the time scale as light variations of $\epsilon$ Ori (see also Buysschaert et al. 2017). The star $\zeta$ Ori A has weak magnetic field and the rotational period of about $6.8 \mathrm{~d}$ (Blazère et al. 2015) and therefore it enables us to study the interaction of atmospheric turbulent motions and magnetic field. Both stars show the line profile variability due to corotating interacting regions (Howarth et al. 1998; Kaper et al. 1999), which are likely unrelated to large-scale magnetic fields (David-Uraz et al. 2014). Because the line-driven wind instability is possibly present in all hot star winds being one of the sources of their X-ray emission (e.g. Antokhin et al. 2008; Nazé 2009), the light variations due to to corotating interacting regions and wind instabilities coexist.

The open cluster NGC 2244 observational run of the CoRoT satellite provides even more promising results. The photometric study of the O9V star HD 46202 (Briquet et al. 2011) revealed multiple frequencies in the range $2-5 \mathrm{~d}^{-1}$ and amplitude of the order of $0.01 \mathrm{mmag}$. This can be explained as a result of wind blanketing due to multiple small-scale wind perturbations. O stars HD 46150, HD 46223, and HD 46966 show small amplitude stochastic variations (Blomme et al. 2011, see also 
Fig. 7) that can be connected to the subphotospheric convection (Aerts \& Rogers 2015). Comparing with Fig. 3 follows that such variability can be caused by a relatively large number of wind perturbations and wind blanketing.

This conclusion is further supported by the comparison of the Fourier spectra (Fig. 8) and their fit parameters in Table 1. The slope parameters $a$ of the spectra based on predicted light curves are close to the theoretical results. From Eq. (8) it follows that the observed light variability is likely caused by a very large number of cones $N \sim 10^{2}-10^{4}$, which is consistent with results of numerical simulations (Sundqvist et al. 2018). Numerical tests using the uncertainties of observed data showed that the Fourier spectrum in Fig. 8 is at least one order of magnitude higher than that of observational noise. Therefore, the Fourier spectra in Fig. 8 should correspond to stellar variations.

\section{Discussion and conclusions}

We simulated the light variability of $O$ stars due to variable wind blanketing modulated by line-driven wind instability. We used the output from hydrodynamical wind simulations and combined the derived time dependence of the mass-loss rate with the dependence of optical flux on the wind mass-loss rate derived from global wind models.

The resulting light curve has amplitude of the order of hundredths of magnitude with a typical time scale of several hours. The amplitude is of the order of millimagnitudes (and lower) when assuming that the wind consists of concentric cones in which the wind behaves independently. Such variability is still observable using high precision photometry.

We compared the derived light curve with light curve of $\epsilon$ Ori obtained using the BRITE satellite and with light curves of O stars from open cluster NGC 2244 obtained by CoRoT satellite. The observed light curves show stochastic variability with amplitude and time scale comparable to that derived from simulations. The light curves of NGC 2244 stars yield Fourier spectra that have the same shape as the Fourier spectra of the predicted light curves and the amplitudes that imply presence of large number $\left(N \sim 10^{2}-10^{4}\right)$ of independent surface patches. The shape of the Fourier spectrum is slightly different for $\epsilon$ Ori, but this may be connected with a narrow range of frequencies available for analysis.

The variability due to the wind blanketing is another source of the light variability in $\mathrm{O}$ stars, which contributes to the light variability due to the pulsation and convection (Aerts \& Rogers 2015; Buysschaert et al. 2015). Consequently, it may be problematic to distinguish the wind blanketing variability from other effects. Ultraviolet observations may solve this problem and test the proposed variability mechanism, because the light variability in the ultraviolet (e.g. around $1300 \AA$ ) should be in the antiphase with visual variability (Krtička 2016). Moreover, there may be stars that are variable only because of the wind blanketing, because only perturbations of surface density and velocity (and no variations of the frequency-integrated radiative flux) are required to trigger this type of variability.

The proposed mechanism requires relatively large mass-loss rate variations. Consequently, we do not expect strong light variations connected with corotating interaction regions (Cranmer \& Owocki 1996; Krtička et al. 2004; Lobel \& Blomme 2008). These regions have been invoked to explain the discrete absorption components commonly observed in the ultraviolet lines of hot stars. The discrete absorption components are connected with velocity plateaus in the wind and require order of magnitude lower mass-loss rate variations than those invoked by wind instabilities (David-Uraz et al. 2017b). Therefore, from Eq. (2) we expect up to millimagnitude variations due to corotating interaction regions.

The light variability due to the wind blanketing is another piece of puzzle to the general picture of variability in $\mathrm{O}$ stars. The subsurface convective motions trigger surface light variations connected with pulsations and variable wind blanketing leading to perturbations that disseminate in the wind initiating the line-driven wind instability.

Acknowledgements. JK acknowledges support by grant GAČR 16-01116S. Based on data collected by the BRITE Constellation satellite mission, designed, built, launched, operated and supported by the Austrian Research Promotion Agency (FFG), the University of Vienna, the Technical University of Graz, the Canadian Space Agency (CSA), the University of Toronto Institute for Aerospace Studies (UTIAS), the Foundation for Polish Science \& Technology (FNiTP MNiSW), and National Science Centre (NCN). The CoRoT space mission, launched on 2006 December 27, was developed and operated by the CNES, with participation of the Science Programs of ESA, ESA's RSSD, Austria, Belgium, Brazil, Germany and Spain.

\section{References}

Abbott, D. C., \& Hummer, D. G. 1985, ApJ, 294, 286

Aerts, C., \& Rogers, T. M. 2015, ApJ, 806, L33

Aerts, C., Puls, J., Godart, M., \& Dupret, M.-A. 2009, A\&A, 508, 409

Aerts, C., Simon-Diaz, S., Bloemen, S., et al. 2017, A\&A, 602, A32

Antokhin, I. I., Rauw, G., Vreux, J.-M., van der Hucht, K. A., \& Brown, J. C. 2008, A\&A, 477, 593

Baade, D., Rivinius, T., Pigulski, A., et al. 2016, A\&A, 588, A56

Blazère, A., Neiner, C., Tkachenko, A., Bouret, J.-C., \& Rivinius, T. 2015, A\&A, 582, A110

Blomme, R., Mahy, L., Catala, C., et al. 2011, A\&A, 533, A4

Bohannan, B., Abbott, D. C., Voels, S. A., \& Hummer, D. G. 1986, ApJ, 308, 728

Briquet, M., Aerts, C., Baglin, A., et al. 2011, A\&A, 527, A112

Burderi, L., Robba, N. R., Guainazzi, M., \& Cusumano, G. 1993, Nuovo Cimento C Geophys. Space Phys. C, 16, 675

Buysschaert, B., Aerts, C., Bloemen, S., et al. 2015, MNRAS, 453, 89

Buysschaert, B., Neiner, C., Richardson, N. D., et al. 2017, A\&A, 602, A91

Cantiello, M., Langer, N., Brott, I., et al. 2009, A\&A, 499, 279

Cranmer, S. R., \& Owocki, S. P. 1996, ApJ, 462, 469

Crowther P. A., Hillier D. J., Evans C. J., et al. 2002, ApJ, 579, 774

Daszyńska-Daszkiewicz, J., Pamyatnykh, A. A., Walczak, P., et al. 2017, MNRAS, 466, 2284

David-Uraz, A., Wade, G. A., Petit, V., et al. 2014, MNRAS, 444, 429

David-Uraz, A., Wade, G., Moffat, A., et al. 2017a, Proc. Pol. Astron. Soc., in press [arXiv: 1711.08394]

David-Uraz, A., Owocki, S. P., Wade, G. A., Sundqvist, J. O., \& Kee, N. D. 2017b, MNRAS, 470, 3672

Davies, B., Vink, J. S., \& Oudmaijer, R. D. 2007, A\&A, 469, 1045

Degroote, P., Briquet, M., Auvergne, M., et al. 2010, A\&A, 519, A38

Derekas, A., Plachy, E., Molnár, L., et al. 2017, MNRAS, 464, 1553

Dessart, L., \& Owocki, S. P. 2002, A\&A, 383, 1113

Feldmeier, A., Puls, J., \& Pauldrach, A. W. A. 1997, A\&A, 322, 878

Feldmeier, A., Oskinova, L., \& Hamann, W.-R. 2003, A\&A, 403, 217

Handler, G., Rybicka, M., Popowicz, A., et al. 2017, MNRAS, 464, 2249

Hedstrom, G. W. 1979, J. Comp. Phys., 30, 222

Howarth, I. D., Townsend, R. H. D., Clayton, M. J., et al. 1998, MNRAS, 296, 949

Hubeny, I., \& Mihalas, D. 2014, Theory of Stellar Atmospheres (Princeton: Princeton University Press)

Jiang, Y.-F., Cantiello, M., Bildsten, L., Quataert, E., \& Blaes, O. 2015, ApJ, 813,74

Kaiser, A., Mochnacki, S., \& Weiss, W. W. 2008, Comm. Asteroseismol., 152, 43

Kaper, L., Henrichs, H. F., Nichols, J. S., \& Telting, J. H. 1999, A\&A, 344, 231

Krtička, J. 2016, A\&A, 594, A75

Krtička, J., \& Kubát, J. 2017, A\&A, 606, A31

Krtička, J., Barrett, R. K., Brown, J. C., \& Owocki, S. P. 2004, A\&A, 417, 1039

Lenz, P., \& Breger, M. 2005, Commun. Asteroseismol., 146, 53

Lobel, A., \& Blomme, R. 2008, ApJ, 678, 408

Lucy, L. B., \& Solomon, P. M. 1970, ApJ, 159, 879

Mahy, L., Gosset, E., Baudin, F., et al. 2011, A\&A, 525, A101 
J. Krtička and A. Feldmeier: Light variations due to the line-driven wind instability and wind blanketing in O stars

Martins, F., Schaerer, D., \& Hillier, D. J. 2005, A\&A, 436, 1049

Nazé, Y. 2009, A\&A, 506, 1055

Nazé, Y., Oskinova, L. M., \& Gosset, E. 2013, ApJ, 763, 143

Oskinova, L. M., Hamann, W.-R., \& Feldmeier, A. 2007, A\&A, 476, 1331

Owocki, S. P. 1991, in Stellar Atmospheres: Beyond Classical Models, eds. L. Crivellari, I. Hubeny, \& D. G. Hummer (Dordrecht: Kluwer), 235

Owocki, S. P., \& Puls, J. 1996, ApJ, 462, 894

Owocki, S. P., \& Rybicki, G. B., 1984, ApJ 284, 337

Owocki, S. P., \& ud-Doula, A. 2004, ApJ, 600, 1004

Owocki, S. P., Castor, J. I., \& Rybicki, G. B. 1988, ApJ, 335, 914

Pablo, H., Richardson, N. D., Moffat, A. F. J., et al. 2015, ApJ, 809, 134

Ramiaramanantsoa, T., Moffat, A. F. J., Harmon, R., et al. 2018, MNRAS, 473, 5532

Reeve, D. C., \& Howarth, I. D. 2016, MNRAS, 456, 1294

Runacres, M. C., \& Owocki, S. P. 2002, A\&A, 381, 1015
Shenar, T., Oskinova, L., Hamann, W.-R., et al. 2015, ApJ, 809, 135

Simón-Díaz, S., Aerts, C., Urbaneja, M. A., et al. 2018, A\&A, 612, A40

Sundqvist, J. O., Puls, J., \& Feldmeier, A. 2010, A\&A, 510, A11

Sundqvist J. O., Puls J., Feldmeier, A., \& Owocki S. P., 2011, A\&A, 528, A6

Sundqvist, J. O., Owocki, S. P., \& Puls, J. 2018, A\&A, 611, A17

Šurlan B., Hamann W.-R., Kubát J., Oskinova L., \& Feldmeier A., 2012, A\&A, 541, A37

Šurlan, B., Hamann, W.-R., Aret, A., et al. 2013, A\&A, 559, A130

Thompson, K. W. 1987, J. Comp. Phys., 68, 1

Thompson, K. W. 1990, J. Comp. Phys., 89, 439

Threlfall, J., De Moortel, I., \& Conlon, T. 2017, Sol. Phys., 292, 165

van Leer, B. 1977, J. Comp. Phys., 23, 276

Weiss, W. W., Rucinski, S. M., Moffat, A. F. J., et al. 2014, PASP, 126, 573

Weiss, W. W., Fröhlich, H.-E., Pigulski, A., et al. 2016, A\&A, 588, A54 